\title{
The Influence of the Layer Sizes on the Conversion Regimes Realizing at Layered Composite Synthesis
}

\author{
Kamila A. Aligozhina ${ }^{1, \text { a) }}$ and Anna G. Knyazeva ${ }^{2,3, \text { b) }}$ \\ ${ }^{1}$ National Research Tomsk State University, Tomsk, 634050, Russia \\ ${ }^{2}$ Institute of Strength Physics and Materials Science SB RAS, Tomsk, 634055, Russia \\ ${ }^{3}$ National Research Tomsk Polytechnic University, Tomsk, 634050, Russia \\ a) Corresponding author: kam.777@mail.ru \\ b) anna-knyazeva@mail.ru
}

\begin{abstract}
The composite materials with different structure find a variety of applications. However, the peculiarities of the propagation of chemical reactions between structural elements during composite material synthesis are poorly studied. This paper suggests a model describing the conjugation of structure composite elements using a solid phase reaction at the ignition from a free surface. It was established that depending on the relation between layer sizes, various conversion regimes between inert materials are observed. The regimes differ in temperature values in the reaction zone, heterogeneities of temperature field and reaction zone thickness. Relation between thermophysical properties and thicknesses of layers can both promote reaction and retard it.
\end{abstract}

Keywords: composite synthesis, material conjugation, exothermal reactions, combustion modes, thermophysical properties, temperature field

\section{INTRODUCTION}

Production of new materials is one of the most important areas determining the development of all sectors of industry, construction, medicine. There is an appreciable progress in the development and production of ceramics, glass and composite materials with various structure elements. In any case, there is a problem on how to form an adequate contact between matrix and inclusions, and between different materials with given properties depending on synthesized material assignment. Similar problem appears when layered composites are formed from interchanging inert materials and exothermic powder mixtures. Reaction proceeds between inert materials with the reaction rate depending on properties and size of layers. Initiation and propagation of the reaction is possible during the consolidation of powders with inert inclusions assisted by electric field or pressure or during the compaction because of local heat release.

Similar situation appears when permanent "metal-metal" and "ceramics-ceramics" bonds are created. In this case, it is called a welding via high temperature synthesis.

Generally, employment of the quickly releasing chemical heat instead of external heating is a promising approach for modern technologies [1]. High temperature gradients observed during the synthesis in solid phase and high rate of the synthesis obstruct the natural experiment with thin physical effects. Mathematical modeling can help here.

Enormous number of papers is devoted to the modeling of the combustion of heterogeneous systems (structurally heterogeneous, layered, mixtures with inclusions). To study thermal physical and kinetic phenomena connecting with difference in properties of materials and presence of interfaces, the paper reporting the combustion of a layered system can be distinguished, for example [2]. The numerical modeling of layered composite synthesis was carried out in [3] with the detailed description of micro wave heating. The review [4] is devoted to multi layered systems.

International Conference on Physical Mesomechanics of Multilevel Systems 2014

AIP Conf. Proc. 1623, 7-10 (2014); doi: 10.1063/1.4901474

(C) 2014 AIP Publishing LLC 978-0-7354-1260-6/\$30.00 


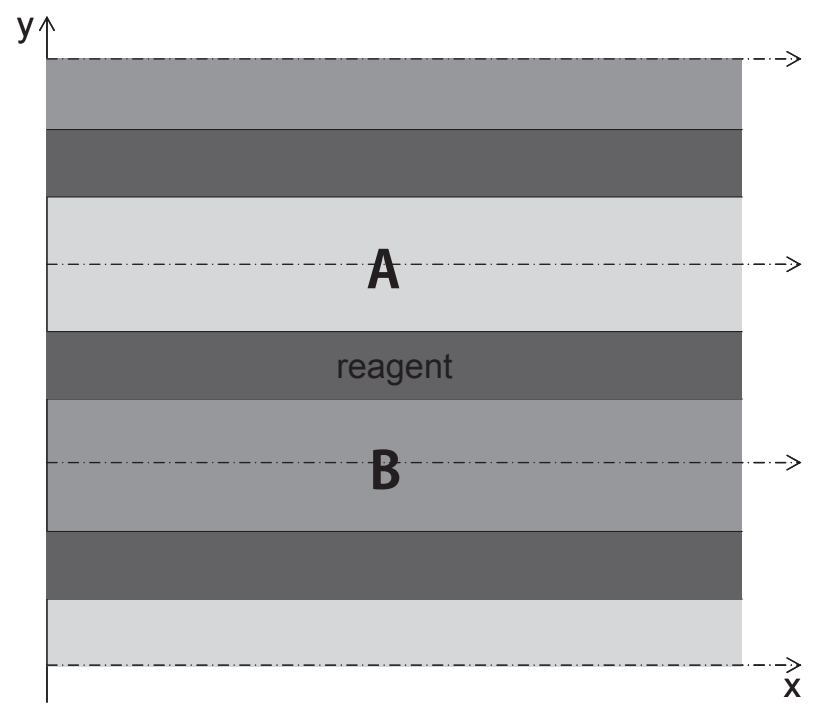

FIGURE 1. The problem statement illustration

This paper studies the problem on the propagation of reaction front between inert layers with different thermalphysical properties.

\section{THE PROBLEM FORMULATION}

The specimen includes decompounded layers of three different materials, including exothermic mixture. Layers are placed in parallel to each other (Fig. 1). Between the layers, an ideal heat contact is considered. In the first approximation, thermophysical properties do not depend on temperature. The chemical reaction in exothermic mixture is described by a simple scheme "reagent-product".

Impulse heating of specimen is carried out from the side of plane $x=0$. Conditions of heat insulation or symmetry for other surfaces remain the same.

Hence, we can speak about a two-dimensional formulation of the problem in the plane $X O Y$.

The problem includes equations of chemical kinetics, thermal conductivity for reagent containing an additional heat source, which corresponds to the heat release due to a chemical reaction, and thermal conductivity equations for inert materials, as well as boundary and initial conditions corresponding to the described situation.

To diminish the number of variables and the amount of necessary numerical calculations we solve the problem in dimensionless form. To transfer to the dimensionless variables, the scales that are typical for the combustion theory were used. The mathematical formulation of the problem is given in [5].

\section{RESULTS AND DISCUSSION}

Numerical implementation of the model was carried out with the help of implicit difference scheme, coordinate splitting and linear double-sweep method.

The result depends on several parameters. Firstly, those are

$$
K_{\lambda A}=\frac{\lambda_{A}}{\lambda}, K_{\lambda B}=\frac{\lambda_{B}}{\lambda}, K_{c A}=\frac{c_{A} \rho_{A}}{c \rho}, K_{c B}=\frac{c_{B} \rho_{B}}{c \rho},
$$

relative thermophysical properties of inert materials and the reagent. Here $\lambda, c, \rho$ are thermal conductivity coefficients, heat capacities and densities of substances, respectively. The values with indexes relate to inert materials $A$ and $B$, respectively; the values without indexes refer to the reagent. 


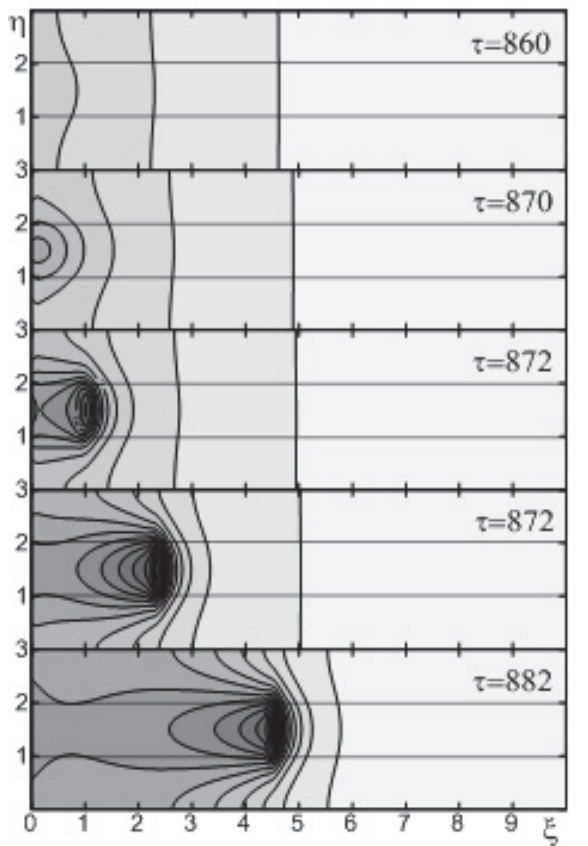

(a)

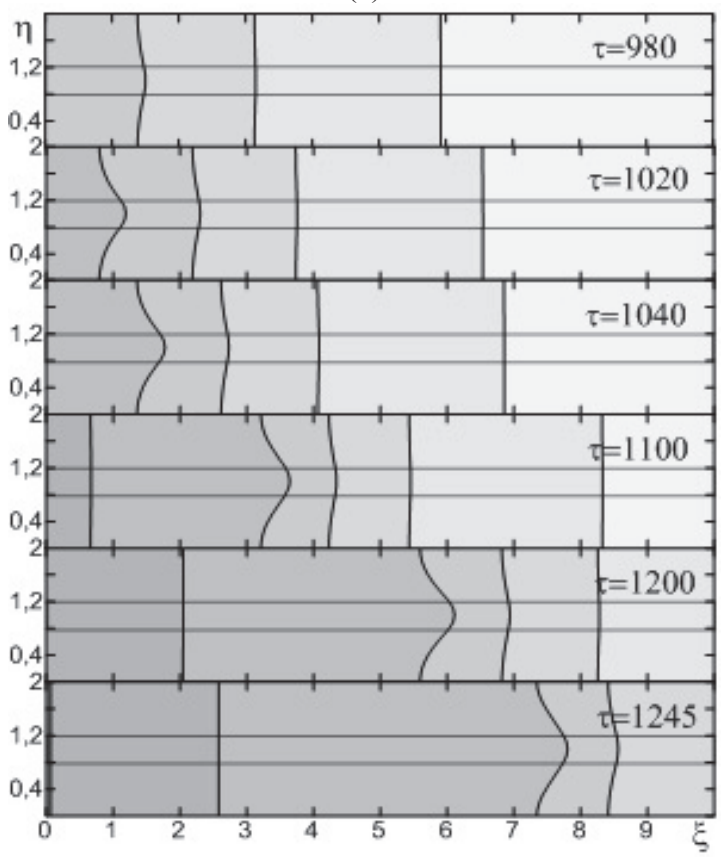

(c)

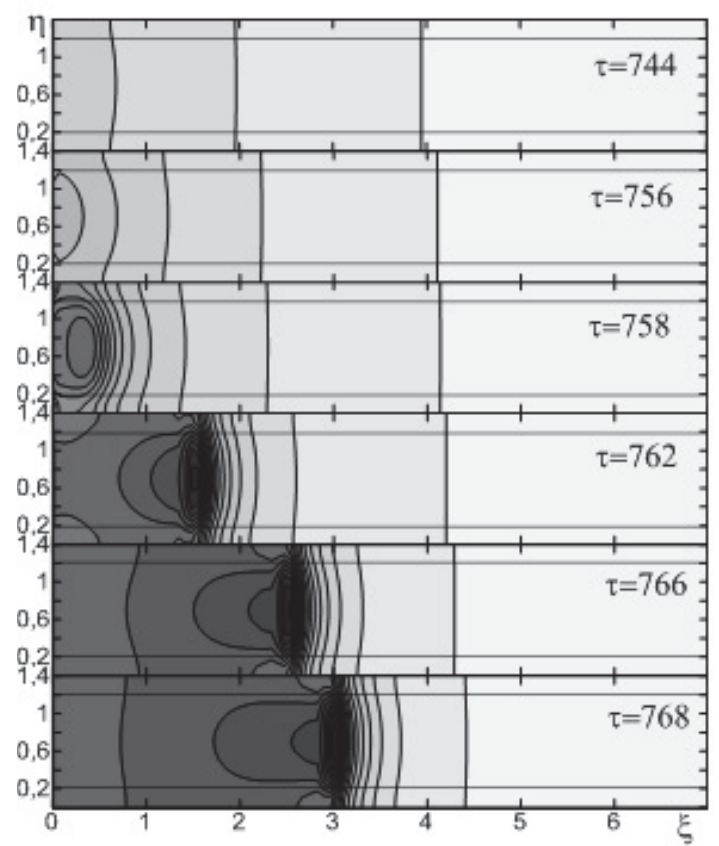

(b)

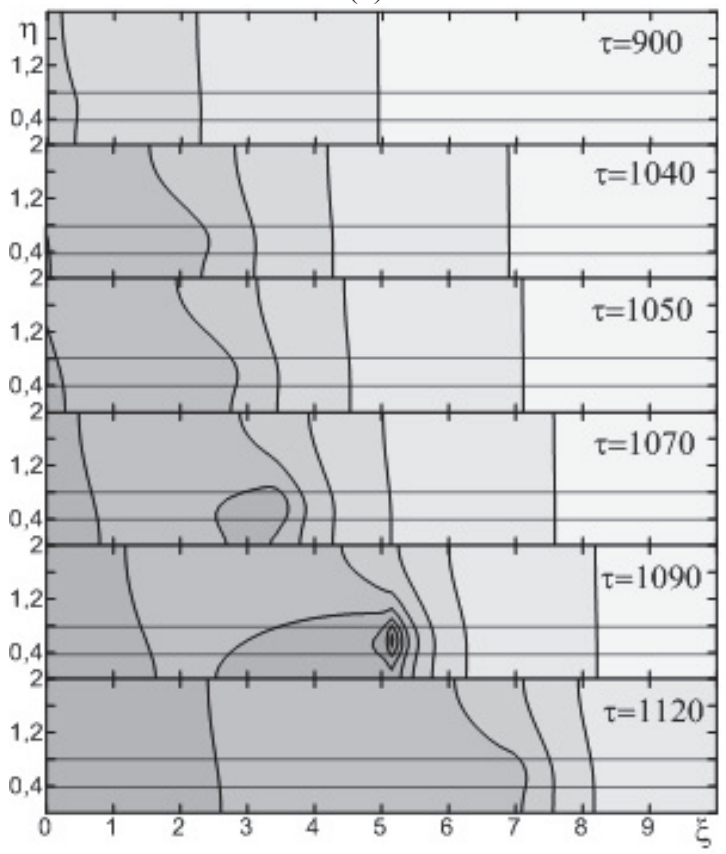

(d)

FIGURE 2. Qualitative presentation of temperature fields for consequent moments of time for various sizes of the layers. Times moments are shown on the figures. Parameters: $\theta_{0}=10, \beta=0.03, \gamma=0.03, F r=20, K_{\lambda A}=K_{\lambda B}=K_{c A}=K_{c B}=1$ 
Secondly, we have the dimensionless complexes:

$$
\theta_{0}=\frac{T_{0}-T_{*}}{R T_{*}^{2}} E, \beta=\frac{R T}{E}, \gamma=\frac{c \rho R T_{*}^{2}}{E Q}, \quad F r=\frac{h^{2}}{x_{T}^{2}},
$$

where $x_{T}=\sqrt{\kappa_{T} t_{*}}$, which is a specific thermal scale, $\kappa_{T}=\lambda /(c \rho)$ is a thermal diffusivity, $h$ is the thickness of reagent layer, $T_{0}$ is initial temperature, $R$ is a universal gas constant, $E, Q$ are formal kinetic parameters for overall chemical reaction. Unlike [5], the thickness of inert layers play a significant role.

Figure 2 illustrates the influence of the ratios of layer sizes on the qualitative behavior of temperature field. In these figures, the darker areas correspond to the higher temperature. Reagent occupies the region between two horizontal lines on the each plot; $\xi, \eta$ are dimensionless coordinates $(\xi=x / h, \eta=y / h)$. All results presented here are obtained for the case when all the layers have identical thermophysical properties.

If inert layers and reagent have equal thickness, they play a role of heat sources for the reaction mixture after external heating and can promote the stable propagation of the reaction front (Fig. 2(a)). The same effect takes place when the inert layers have thickness less then reagent one (Fig. 2(b)). However, the reaction starts earlier then in previous case, because heating of thin inert materials needs less heat. In this case, higher temperature gradients are observed, which is undesirable because thermomechanical stresses can appear (not calculated here).

Another character of the reaction front propagation is demonstrated in Fig. 2(c, d).

If the thicknesses of inert materials are different, the reaction front is bended significant. Reaction propagates in asymmetric conditions. In Fig. 2(d), the thickness of the top layer is three times larger than thickness of the reagent and the bottom layer has the same thickness as the reagent. In this case, the reaction retardation connects with heat losses to top layer of composite.

If inert structure element materials have the thickness two times larger than the reagent one, they promote the heat loss from the reaction zone, and hence they significantly retard the process (Fig. 2(c)). In this case, finite heat storage obtained during the impulse action and chemical heat release would be not enough for the self-sustaining mode of reaction propagation.

The phenomena connected with the imperfect contact between substances play a significant role in the formation of synthesized composite properties that can be taken into account in the model. Model can be augmented by subtasks for the simulation of the forming transient zone between materials and for the calculation of stresses in the synthesized composite.

\section{SUMMARY}

In this work, the examples of temperature fields were presented for various ratios of sizes between inert materials and reagent. It was shown that inert materials can both promote and retard the reaction development. Therefore, it is necessary to thoroughly choose the size of layers (or structure elements) correspondingly to the size of the area occupied by the reagent, when the synthesis of a composite is carried out using solid phase reaction.

The work is supported by TPU grant No. VIU_IFVT_85_2014.

\section{REFERENCES}

1. A. G. Merzhanov, Solid Flame Combustion (Izd. ISMAN, Chernogolovka, 2000).

2. O. N. Kirsanov, Russ. J. Appl. Chem. 78(11), 1779 (2005).

3. S. Ghafurian, S. H. Seydein, M. R. Aboutabeli, and M. Reza Afshar, Iran J. Mater. Sci. Eng. 8(3), 8 (2011).

4. Krishenik, S. A. Rogachev, and K. G. Shkadinsky, Int. J. Self-Propag. High Temp. Synth. 21(2), 83 (2012).

5. K. A. Aligozhina and A. G. Knyazeva, Russ. Phys. J. 56, 34 (2013). 